\title{
MOVIMIENTO NORMAL DE LA FALLA PAPOSO, ZONA DE FALLA ATACAMA, EN EL MIOCENO, CHILE
}

\section{RESUMEN}

La Zona de Falla Atacama, que recorre la Cordillera de la Costa del norte de Chile a lo largo de unos $1.000 \mathrm{~km}$, está formada por una serie de fallas de rumbo general norte-sur. La falla principal del sistema al norte de Paposo (2400'$24^{\circ} 45^{\prime}$ S), denominada aquí Falla Paposo, presenta un escarpe cara al este, de hasta unos $300 \mathrm{~m}$ de altura, producto del alzamiento relativo del bloque occidental.

El escarpe de la Falla Paposo produjo el entrampamiento de un sistema de drenajes locales, de edad miocena o más antigua, de dirección general este-oeste, lo que originó la depositación de "playas" o "lagos secos" en estas cuencas cerradas por el escarpe. Eliminando la topografía que corresponde a los rasgos del escarpe, se verifica que los ejes del drenaje atraviesan la falla sin desviación lateral alguna, indicando que el movimiento generador del escarpe de falla fue vertical, sin componente en el rumbo.

El movimiento vertical se produjo con posterioridad a los $19 \mathrm{Ma}$, edad de un flujo piroclástico "colgado" en el escarpe y depositado desde el este, antes de la existencia del relieve de falla. Un nivel de cenizas datado en 5,5 Ma, está interestratificado en conos de deyección producto de la erosión del escarpe, indicando su presencia ya en esa época. El período de actividad vertical de la Falla Paposo se ubica en el intervalo de tiempo enire los 19 y 5,5 Ma (Mioceno InferiorMioceno Superior). En la zona no se han hallado evidencias de actividad de la Falla Paposo, con posterioridad al Mioceno.

Palabras claves: Falla Atacama, Movimiento normal, Dataciones K-Ar, Mioceno, Norte de Chile.

\section{ABSTRACT}

The Atacama Fault Zone, located in the Coastal Range of northern Chile, is composed of a system of parallel trending faults with a general north-south arrangement. In the area north of Paposo (24 $\left.00^{\prime}-24^{\circ} 45^{\prime} \mathrm{S}\right)$, the most important fault of the system is here named Paposo Fault and has produced an east-facing scarp up to $300 \mathrm{~m}$ high, with upward movement of the western block.

The Paposo Fault scarp interrupts a preexisting drainage pattern of local origin, and Miocene or older age, forcing the deposition of "playas" or "dry lakes". By eliminating the topographic features produced by the scarp it is seen that the drainage axis crosses the fault zone with no lateral displacement, a clear indication that the scarp generating movement was vertical, with no along-strike component.

The vertical activity of the Paposo Fault occurred after the deposition of a $19 \mathrm{Ma}$ pyroclastic flow located to the west of the scarp. If the scarp would have been there at the moment of the east coming flow, it is unlikely that it could have reached its current position. An ashfall deposit, dated in $5.5 \mathrm{Ma}$ is interbedded in an alluvial fan produced by the erosion of the scarp, indicating that it already existed by that time. The vertical activity of the Paposo Fault is then located somewhere between 19 and $5.5 \mathrm{Ma}$ (Lower-Upper Miocene). No evidence of post-Miocene activity of the Paposo Fault has been detected in the study area.

Key words: Atacama fault, Normal movement, K-Ar dating, Miocene, Northern Chile.

\section{INTRODUCCION}

La Zona de Falla Atacama es una de las estructuras más importantes de la Cordiliera de la Costa del Norte Grande de Chile. Se extiende desde la latitud de lquique, por el norte $\left(20^{\circ} \mathrm{S}\right)$, hasta la latitud de Copiapó, por el sur ( $\left.27^{\circ} \mathrm{S}\right)$, con una longitud del orden de los $800 \mathrm{~km}$. Diversos autores han estudiado esta zona de falla a lo largo de su extensión (St. Amand y Allen, 1960; Arabasz, 1971; Naranjo 
et al., 1984; Hervé, 1987).

La Zona de Falla Atacama se subdividió en tres segmentos principales (Arabasz, 1971): uno de Antofagasta al norte, otro entre Antofagasta y Taltal, y un tercero al sur de Taltal. Las observaciones entregadas en este artículo se efectuaron en el tramo intermedio, más precisamente, en la zona entre Paposo, por el sur, y la latitud de Caleta El Cobre, por el norte, entre los $24^{\circ} 00^{\prime}$ y $24^{\circ} 45^{\prime} \mathrm{S}$, aproximadamente. El segmento principal de la Zona de Falla Atacama, en este tramo, se denomina aquí Falla Paposo (Fig. 1).

La Falla Paposo se caracteriza por presentar, en superficie, un escarpe de falla de hasta unos $300 \mathrm{~m}$ de altura. El escarpe mira al este, exhibe el desarrollo de facetas triangulares y se ha generado por el alzamiento relativo del bloque occidental. Tiene una longitud cercana a los $80 \mathrm{~km}$ y su traza es ligeramente cóncava hacia el oeste.

Estudios en distintos segmentos de la Zona de Falla Atacama, han demostrado la existencia de actividad transcurrente en épocas pre-terciarias (Arabasz,1971; Naranjo et al., 1984; Hervé, 1987). Menos atención se le ha prestado a los movimientos tardíos de la falla, que son los que se manifiestan en los rasgos morfológicos espectaculares que presenta esta estructura en la zona del presente estudio.

El levantamiento geológico del sector costero de la Hoja Aguas Blancas (Programa Carta Geológica de Chile, escala 1:250.000, Servicio Nacional de Geología y Minería) ha permitido realizar observaciones a lo largo de la traza de la Falla Atacama entre los paralelos $24^{\circ}$ y $25^{\circ} \mathrm{S}$, es decir, en la Falla Paposo. Esta falla está acompañada por una serie de fallas asociadas, de las que sobresalen las fallas Izcuña y Caleta Coloso (Arabasz, 1971)(Fig. 1).

La información que aquí se presenta está enfocada a dilucidar el tipo de movimiento que ha afectado a la Falla Paposo, en sus últimos episodios de actividad. Se analiza el efecto que éste ha producido en redes de drenaje miocenas o más antiguas. También se estudia la cronología de estos movimientos basada en la datación K-Ar de algunos flujos piroclásticos y depósitos de ceniza existentes en el área.

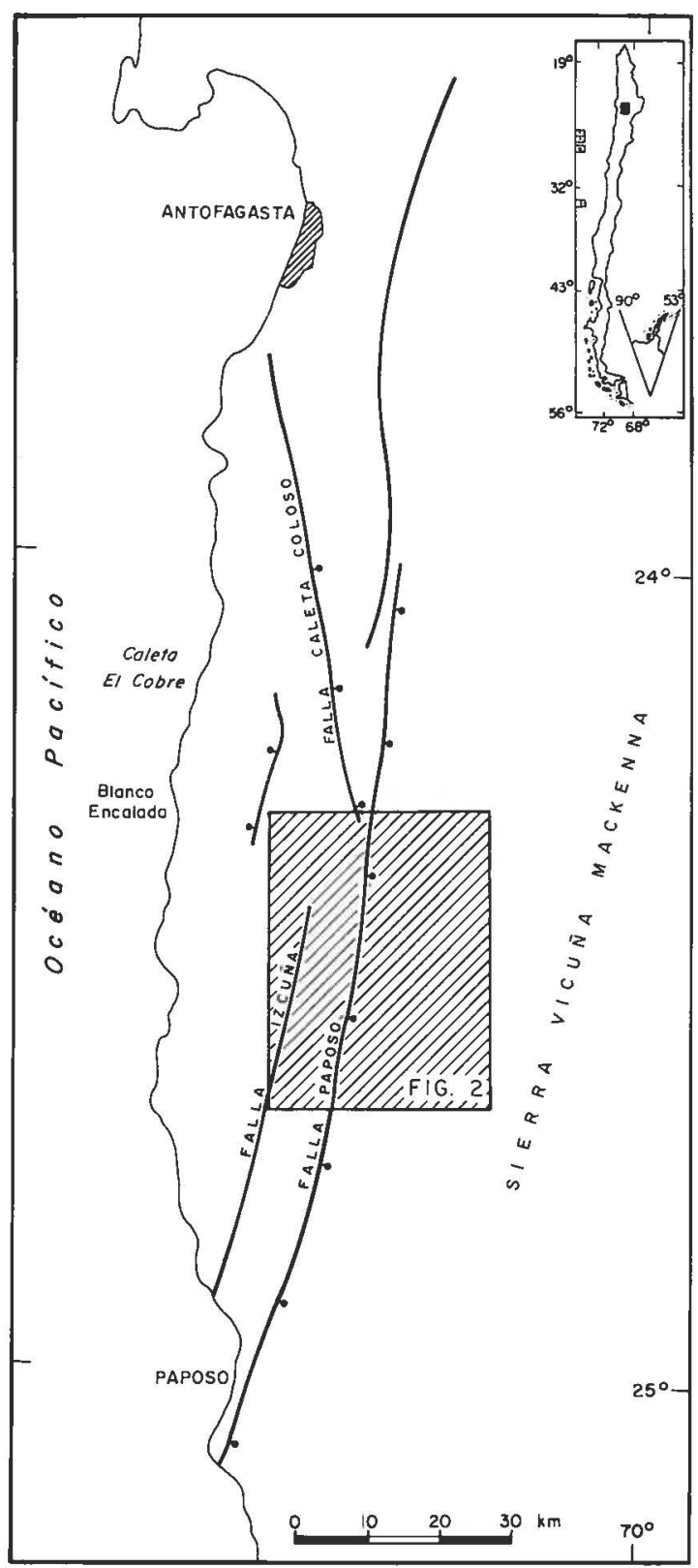

FIG. 1. Ubicación de las fallas principales de la Zona de Falla Atacama, en la región entre Paposo y Antofagasta. Se destacan la Falla Paposo y la zona representada en la figura 2.

\section{LA FALLA PAPOSO}

La Falla Paposo está acompañada por una serie de fallas aproximadamente paralelas, con expre- sión morfológica subordinada. Afectan a rocas plutónicas de edades jurásicas y cretácicas, que 
constituyen la mayoría de los afloramientos de la Cordillera de la Costa en el sector. Algunas fallas no muestran escarpes y se manifiestan sólo como discontinuidades litológicas, a menudo, acompañadas por rocas de falla, probablemente debido a que han permanecido selladas desde los episodios transcurrentes del Cretácico que las formaron (Naranjo, et al., 1984; Hervé, 1987).

En este sector de la Zona de Falla Atacama se ha mostrado la existencia de movimientos de rum- bo sinistrales, con desplazamientos de hasta 34 $\mathrm{km}$, de un plutón a lo largo del Sistema de Falla lzcuña (Hervé, 1987). La mayor parte de las fallas que muestran escarpes presentan un alzamiento relativo del bloque occidental, de hasta unos 300 $\mathrm{m}$. La única falla documentada con escarpe, producto del alzamiento del bloque oriental, se ubica al interior de Blanco Encalada-Quebrada El Cobre (Fig. 1).

\section{MORFOLOGIA Y MODELOS DE DRENAJE}

El segmento de la Zona de Falla Atacama, aquí estudiado, está limitado al norte y al sur por profundas quebradas de dirección general este-oeste, que desaguan en megahoyas, en el sentido de $\mathrm{Na}-$ ranjo (1987). En el sector central de la Falla Paposo, que corresponde también al de mayor altura, se ha reconocido la existencia de una morfología relicta, que precede la formación de estas megahoyas y que, aparentemente, no ha sido reactivada junto con ellas. Se trata de un modelo de drenaje local, con cabeceras en el flanco occidental de Sierra Vicuña Mackenna (Fig. 1), que drenaron en dirección al oeste. En su límite occidental, actualmente, estos valles están "colgados" en el acantilado costero. En sus tramos intermedios, se presentan seccionados por el escarpe de la Falla Paposo, que controla la presencia de "playas" o "lagos secos", al entrampar algunos de estos drenajes.

En el modelo de drenaje local en referencia, se distinguen al menos cuatro zonas, considerando las características del relieve. De este a oeste, éstas son: zona de Sierra Vicuña Mackenna, con un drenaje profundo; zona de Pampa Remiendos, con relieves suaves; zona al oeste de Falla Paposo, con drenajes profundos similares a la primera zona $y$, finalmente, la zona del escarpe o del acantilado costero, con un relieve extraordinariamente abrupto y profundo. Esta última zona corresponde, indudablemente, a un relieve más joven. Las diferencias en la morfología de las tres zonas alejadas de la costa, se interpretan como producto del control litológico sobre el relieve. En efecto, tanto la zona más oriental como la occidental, ambas con drenajes profundos, están labradas en gabroides, mientras que la zona intermedia de Pampa Remiendos, con relieves suaves, se desarrolló en granitos y granodioritas.

El análisis cronológico de rocas piroclásticas indicó que este relieve labrado en rocas plutónicas es anterior a los $19 \mathrm{Ma}$ (Tabla 1). En efecto, en las nacientes de la actual Quebrada Remiendos, al oeste de Falla Paposo, la datación de un flujo piroclástico, que se emplazó por el lecho de la quebrada, indicó esa edad. Se considera ésta una edad mínima para el modelado del paisaje en referencia, el que se ha mantenido casi sin modificaciones hasta el presente.

TABLA 1. RESULTADOS ANALITICOS DE LAS DATACIONES K-Ar

\begin{tabular}{|llcccc|}
\hline Muestra & Material & $\begin{array}{c}\text { Potasio } \\
\%\end{array}$ & $\begin{array}{c}\text { 40Ar rad. } \\
\mathbf{n l} / \mathbf{g} \text { (STP) }\end{array}$ & $\begin{array}{c}\text { Ar Atm. } \\
\%\end{array}$ & $\begin{array}{c}\text { Edad y Error } \\
\text { Ma (2 } \sigma)\end{array}$ \\
\hline HAB-775 & $\begin{array}{l}\text { Biotita de flujo } \\
\text { piroclástico } \\
\text { Biotita de depósito } \\
\text { HAB-663 ceniza }\end{array}$ & 5,432 & 4,036 & 72 & $19,0 \pm 1,2$ \\
\hline
\end{tabular}

Las dataciones fueron hechas en el Laboratorio de Geocronología K-Ar del Servicio Nacional de Geologla y Minería.

Constantes de Steiger y Jäger, 1977. 
a

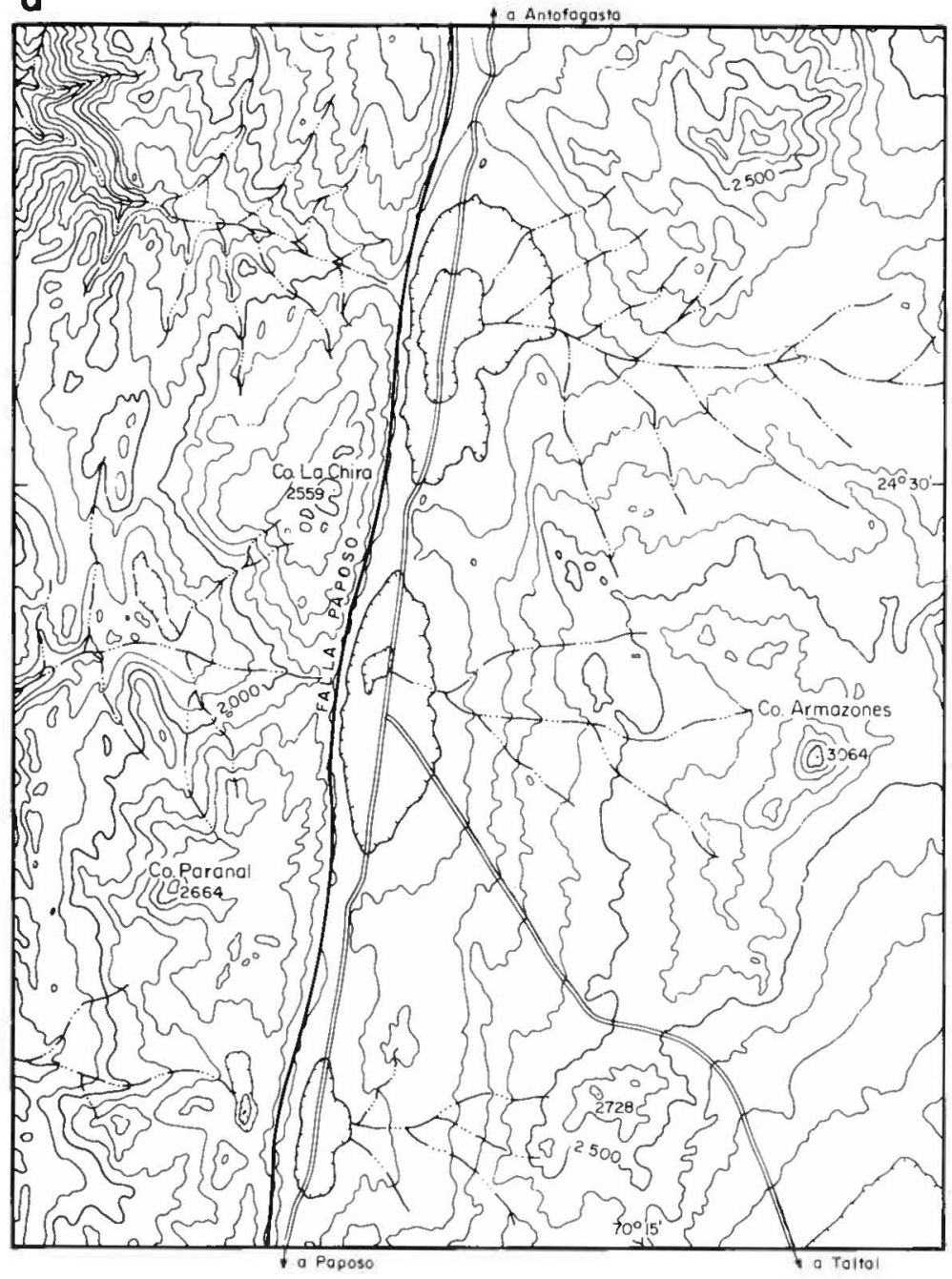

b

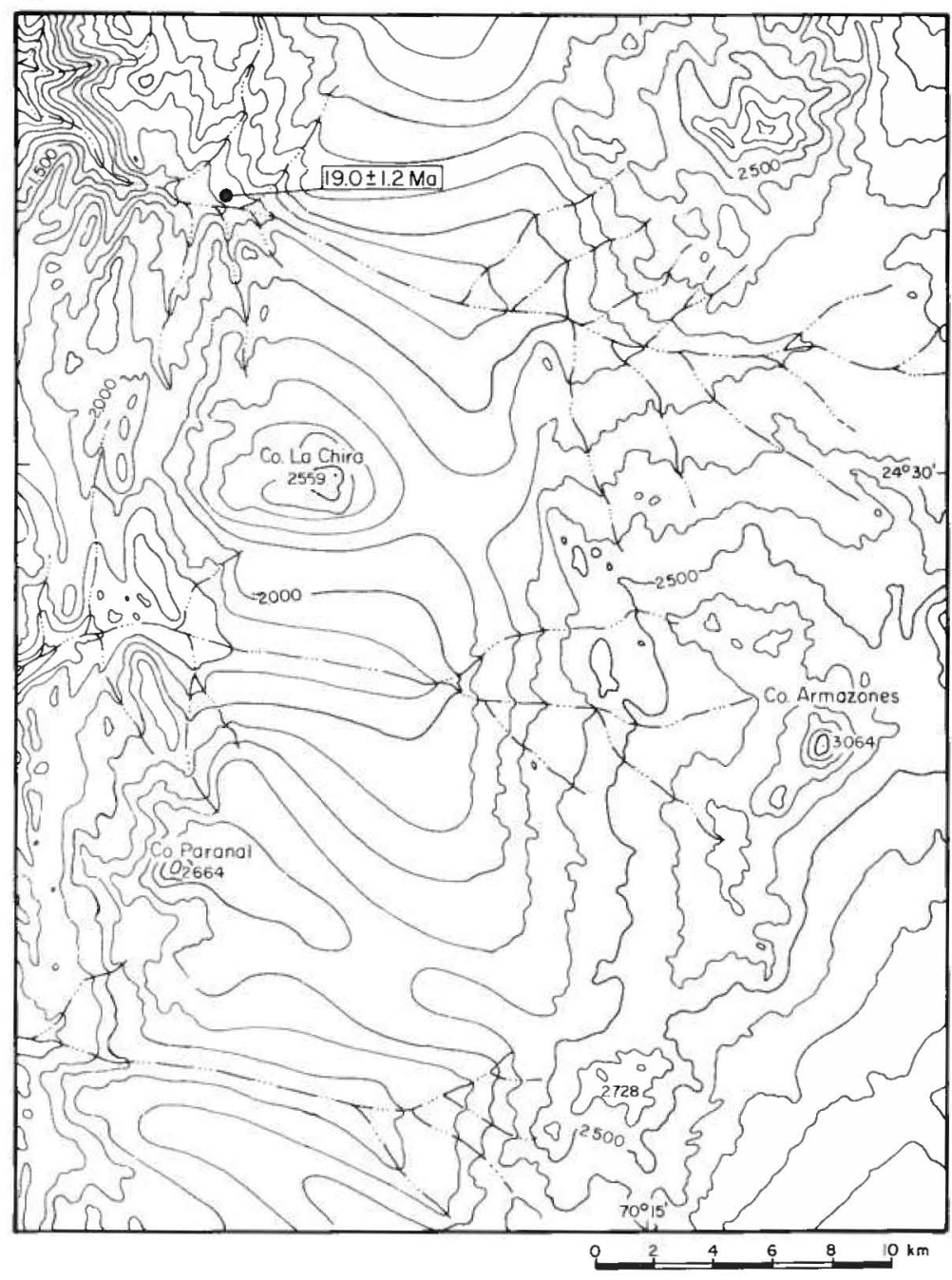
existente antes del movimiento vertical de la falla, lograda eliminando $300 \mathrm{~m}$ de curvas de nivel en el escarpe. Nótese la continuidad de los ejes del drenaje al atravesar la falla. Se destaca la ubicación del flujio piroclástico datado. 


\section{EFECTOS DE LA FALLA PAPOSO EN EL DRENAJE (¿PRE?)MIOCENO}

La Falla Paposo afectó al drenaje local este-oeste descrito, debido a que, a lo largo de su traza, se produjo un escarpe de unos $300 \mathrm{~m}$ de altura, con alzamiento del bloque occidental. Este escarpe capturó el drenaje, a consecuencia de lo cual, se produjeron "playas" o "lagunas secas" debidas a la depositación de materiales de grano fino, transportados en suspensión por cursos fluviales, que quedaron embalsados por el escarpe.

Con el fin de analizar el efecto que produjo el movimiento de la Falla Paposo en la red de drenaje, se puede utilizar el simple expediente de eliminar, en una carta topográfica adecuada, todas aquellas curvas de nivel que representen la morfología del escarpe de falla, con el fin de restituir el relieve existente antes de la formación de éste. Se obtiene así una serie de drenajes, cuyos ejes atraviesan, sin desviación alguna, la traza de la falla. Se desprende, entonces, que el movimiento de la Falla Paposo, que generó el escarpe, fue exclusivamente de carácter normal, sin componentes de rumbo (Fig. 2).

\section{EDAD DEL MOVIMIENTO NORMAL}

La edad del movimiento normal que afectó a la Falla Paposo, puede ser acotada sobre la base de análisis de edades radiométricas K-Ar en biotita de flujos piroclásticos y de depósitos de caída de cenizas, que afloran en diversos sectores del área. El movimiento habría sido posterior a los $19 \mathrm{Ma}$ (Tabla 1) que es la edad del flujo piroclástico que sella el drenaje, ya que, por tratarse de un flujo cuya fuente se habría localizado bastante lejos hacia el este, es improbable que hubiera remontado el escarpe de falla de haber estado éste presente. La edad mínima del movimiento está dada por una edad de 5,5 Ma (Tabla 1) en biotitas de un depósito de ceniza, situado al este del escarpe y que está inte- restratificado en conos de deyección producto de la erosión del escarpe de falla.

Se concluye, entonces, que la actividad de carácter normal de la Falla Paposo se verificó en un intervalo entre los 19 y 5,5 Ma, es decir, entre el Mioceno Inferior medio y el Mioceno Superior (Escala del Tiempo de Harland et al., 1982). Un intervalo similar de edad para la última actividad de la Falla Atacama ha sido propuesto por Naranjo (1987), basado en el estudio de la morfología y de las redes de drenaje. No se han encontrado evidencias que indiquen actividad de la Falla Paposo con posterioridad al Mioceno, por lo que se estima que está sellada desde esa época.

\section{CONCLUSIONES}

- La Falla Paposo desarrolló una actividad de carácter vertical, sin componente horizontal, con alzamiento relativo del bloque occidental, afectando a redes de drenaje miocenas o más antiguas.

- Se sugiere que la actividad de carácter normal de la Falla Paposo tuvo lugar entre los 19 y 5,5 Ma, es decir entre el Mioceno Inferior medio y el Mioceno Superior. Se estima que éste representa el último movimiento de la falla, la que estaría sellada desde entonces.

\section{AGRADECIMIENTOS}

Este trabajo forma parte del Proyecto No. 202, Megafalla de Sudamérica, correspondiente al Pro- grama Internacional de Correlación Geológica.

\section{REFERENCIAS}

ARABASZ, W. J. 1971. Geological and Geophysical studies of the Atacama Fault Zone in Northern Chile. Ph. D.
Thesis. California Institute of Technology, 264 p. Pasadena, USA. 
HERVE, M. 1987. Movimiento sinistral en el Cretácico Inferior en la Zona de Falla Atacama al norte de Paposo $\left(24^{\circ}\right)$, Chile. Revista Geológica de Chile, No. 31, p. $37-$ 42. Este volumen.

HARLAND, W.B.; COX, A.; LEWELLYN, P.G.; PICKTON, C.A.G.; SMITH, A.G.M.; WALTERS, R. 1982. A geological time scale. University of Cambridge, Cambridge University Press, 1 table.

NARANJO, J.A. 1987. Interpretación de la actividad cenozoica superior a lo largo de la Zona de Falla Atacama, Norte de Chile. Revista Geológica de Chile, No. 31, p. 43-55. Este volumen.
NARANJO, J.A.; HERVE, F., PRIETO, X.; MUNIZAGA, $F$. 1984. Actividad cretácica de la Falla Atacama al este de Chañaral: Milonitización y plutonismo. Comunicaciones, No. 34, p. 57-66.

St. AMAND, P.; ALLEN, C.R. 1960. Strike-slip faulting in Northern Chile. Geological Society of America, Bulletin [Abstracts], Vol. 71, p. 1965.

STEIGER, R.H.; JÄGER, E. 1977. Subcommission in Geochronology: Conventions on the use of Decay constants in Geo and Cosmochronology. Earth and Planetary Science. Letters, Vol. 36, p. 359-362. 